\title{
Fabrication of Paper-based Microfluidic Devices Using a Laser Beam Scanning Technique
}

\author{
Dang Huy HieP, * Yuta Tanaka,* Hiroki Matsubara, ** and Shoji IshizaKa**† \\ *Department of Chemistry, Graduate School of Science, Hiroshima University, 1-3-1 Kagamiyama, \\ Higashi-Hiroshima 739-8526, Japan \\ **Graduate School of Advanced Science and Engineering, Hiroshima University, 1-3-1 Kagamiyama, \\ Higashi-Hiroshima 739-8526, Japan
}

\begin{abstract}
This paper describes a novel method for fabricating paper-based microfluidic devices using a laser beam scanning technique. Cellulose chromatography papers were treated with octadecyltrichlorosilane (OTS) to make them entirely hydrophobic. A photoacid generator (CPI-410S) was soaked into the paper, and irradiated with a 405-nm laser beam to induce acid generating reactions. Since the silyl ether bond between cellulose and OTS was cleaved by the hydrolysis reaction, the photo-irradiated area changed to hydrophilic. By scanning the laser beam using a Galvo mirror system, arbitrary shaped hydrophilic patterns were successfully created on the paper in $50 \mu \mathrm{m}$ resolution. To the best of our knowledge, this is the first report on the fabrication of hydrophilic channels on the OTS-treated paper using photo-induced acid generation processes coupled with the laser beam scanning technique. Quantification of nitrite was demonstrated with the paper device made by this method.
\end{abstract}

Keywords Paper-based microfluidic device, photoacid generator, laser beam scanning technique

(Received June 5, 2020; Accepted June 15, 2020; Advance Publication Released Online by J-STAGE June 19, 2020)

\section{Introduction}

Paper-based microfluidic analytical devices have been attracting increasing attention as a handy, low-cost analytical tool. Since Whitesides's group reported the modern system for the paperbased microfluidic device fabricated by photolithography in $2007,{ }^{1}$ many methods of creating microchannels on paper have been proposed so far. Now a large variety of fabrication techniques are available. ${ }^{2-5}$ Among them, the method using a laser beam has an advantage that microchannels can be directly created on paper without using a photomask. There are two types of strategies to create microchannels on paper using a laser beam. One is to fabricate hydrophobic barriers on hydrophilic paper by photo-polymerization..$^{6,7}$ The other is to fabricate hydrophilic channels on hydrophobic paper by laserirradiation. ${ }^{8} \quad$ Ziaie et al. reported a method fabricating hydrophilic patterns on hydrophobic paper using a computercontrolled $\mathrm{CO}_{2}$ laser. ${ }^{8}$ They used parchment paper, which is hydrophobic because its surface is coated with silicone. When parchment paper was irradiated with the $\mathrm{CO}_{2}$ laser, the laserirradiated part became hydrophilic because the changes in morphology and chemical composition of the surface occurred due to the melting and re-solidification of silicone accompanying the inclusion of oxygen in the atmosphere. Therefore, this method is useful for fabricating hydrophilic channels in paper having any coating materials on the surface. Herein, we propose

$\dagger$ To whom correspondence should be addressed.

E-mail: ishizaka@hiroshima-u.ac.jp a new approach to fabricate hydrophilic channels on the hydrophobic paper using a laser beam scanning technique.

Recently, alkylsilane coupling reagents have been used to modify cellulose based paper from hydrophilic to hydrophobic. ${ }^{9-14}$ Since alkylsilanes are degraded by ozone under deep UV light irradiation, He et al. succeeded in creating hydrophilic channels on the hydrophobic paper, which was made of cellulose fibers chemically coupled with octadecyltrichlorosilane (OTS), by irradiating deep UV light through a photomask. Furthermore, cellulose silyl ethers can be cleaved by acid treatment. Photoacid generators are organic compounds that can generate protons upon photo-irradiation. Spirk et al. reported that desilylation of trimethylsilyl cellulose was induced by UV light irradiation in the presence of $N$-hydroxynaphthalimide as a photoacid generator. ${ }^{15}$ Therefore, it should be possible to fabricate hydrophilic channels directly on the alkylsilane-treated paper by irradiating a laser beam in the presence of the photoacid generator. In this study, we used a derivative of triarylsulfonium salts (CPI-410S) as a photoacid generator, which is used as a photoinitiator for cationic polymerization. ${ }^{16}$ By scanning a 405-nm laser beam using a Galvo mirror system, arbitrary shaped hydrophilic patterns were successfully created on the OTS-treated paper. To the best of our knowledge, this is the first report on the fabrication of hydrophilic channels on the OTS-treated paper using photo-induced acid generation processes coupled with the laser beam scanning technique. Furthermore, quantification of nitrite was demonstrated with the paper device made by this method. 

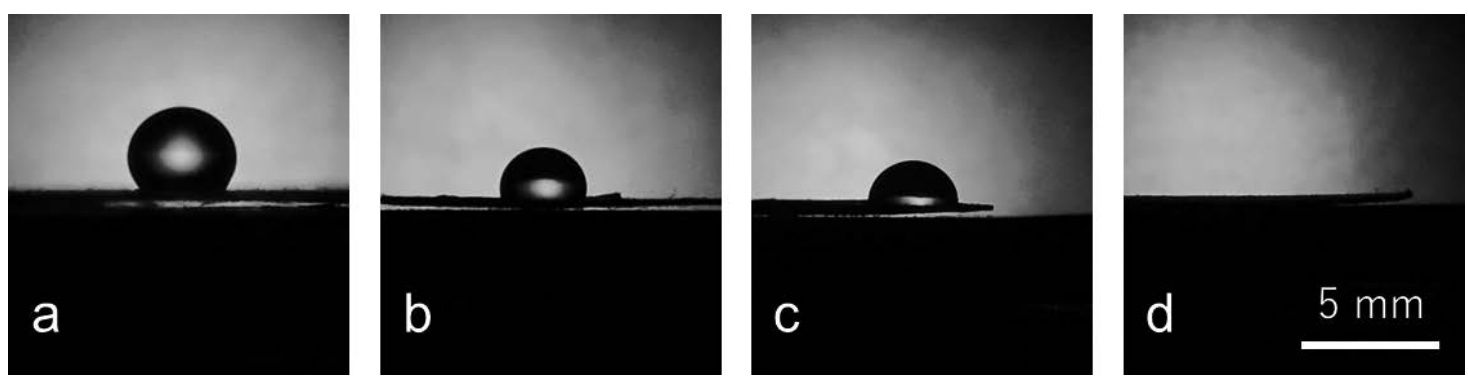

Fig. 1 The contact angle of water droplets on the OTS-treated paper in the presence of CPI-410S.

The paper was illuminated with a xenon lamp for (a) 0 , (b) 20, (c) 40, and (d) $50 \mathrm{~min}$, respectively.
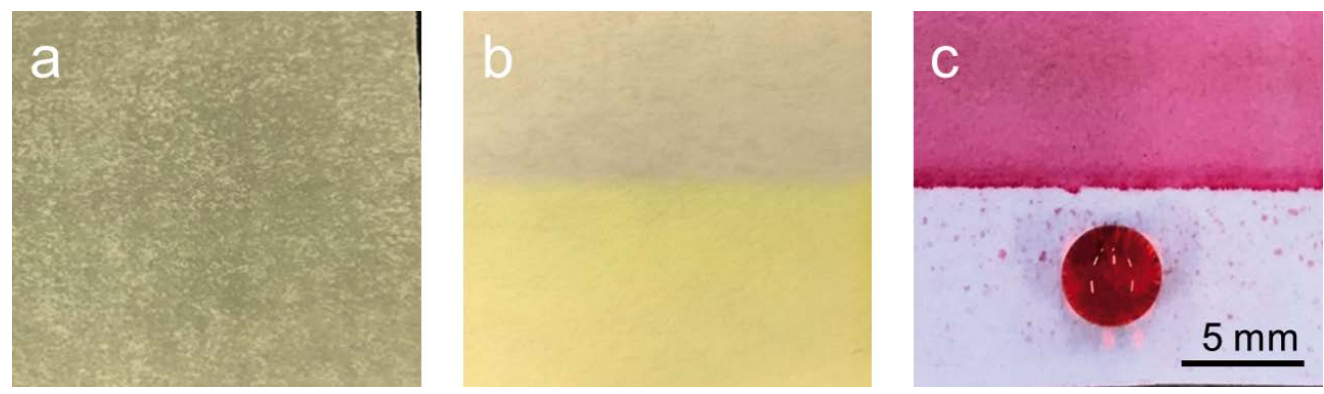

Fig. 2 The images display photographs of the OTS-treated paper, (a) immediately after being removed from DMSO solution of CPI-410S, (b) after photoirradiation for $50 \mathrm{~min}$, and (c) after rinsing with chloroform/DMSO and aqueous droplets containing Acid Red 1 were dropped on the upper part and the lower part.

\section{Experimental}

CPI-410S was supplied by San-Apro Ltd. and used without further purification. The structure of CPI-410S is shown in the Supporting Information, Fig. S1. OTS and Acid Red 1 were purchased from Sigma Aldrich-Merck Co. Dimethyl sulfoxide, chloroform, $n$-hexane, and ethanol were purchased from Wako Pure Chemicals Co. Ltd. and used without further purification. Water was purified by reverse osmosis and deionization prior to use (Merck Millipore, Milli-Q Integral 3). Cellulose chromatography papers (Advantec Co., Ltd.) were immersed in $0.1 \%$ (v/v) OTS $n$-hexane solution for $5 \mathrm{~min}$ at room temperature. The papers removed from the OTS solution were rinsed with $n$-hexane and ethanol and dried in air. Subsequently, the OTStreated papers were immersed in chloroform and 5\% (w/w) CPI410S DMSO solution for 1 and 2 min, respectively. A diode laser $(405 \mathrm{~nm}, 300 \mathrm{~mW}$, Changchun New Industries Optoelectronics Technology Co., Ltd., MDL-III-405-300) and a xenon lamp (150 W, Hamamatsu photonics K.K., L2274) were used as an excitation light source for CPI-410S. A Galvo mirror system (Thorlabs, Inc., GVS002) was used to scan the laser beam.

\section{Results and Discussion}

The contact angle of water droplets on the OTS-treated paper under photoirradiation

In the present study, hydrophilic-hydrophobic patterns on papers were created by two steps. First, cellulose chromatography papers were treated with OTS to make them entirely hydrophobic. Second, hydrophilic patterns were created by the hydrolysis of the silyl ether bond between cellulose and OTS, which was triggered by the photoinduced acid generation reaction of CPI$410 \mathrm{~S}$. In order to confirm that the CPI-410S can remove OTS in the paper under photoirradiation, the contact angle of water droplets on the OTS-treated paper was investigated as shown in Fig. 1. In this experiment, in order to irradiate the entire surface of the paper with light, UV-visible light from a xenon lamp was used instead of the 405-nm laser beam. Before shining light on the paper, the contact angle of the water droplet was approximately $137^{\circ}$ (Fig. 1(a)). When the paper was irradiated with light in the presence of CPI-410S, the contact angle decreased with an increase of irradiation time (Figs. 1(b) and 1(c)). When the light irradiation time was $50 \mathrm{~min}$, the contact angle of the water droplet became $0^{\circ}$ (Fig. 1(d)). This result clearly shows that the hydrophobic paper has turned into a completely hydrophilic one. Generally, triarylsulfonium salts are used as a photoinitiator of cationic polymerization, but we demonstrated for the first time that they can also be used for a photoinduced hydrolysis reaction in the paper treated with OTS.

Hydrophilic-hydrophobic contrast in the photo-irradiated OTStreated paper

To fabricate hydrophilic channels on OTS-treated papers, it is necessary that only the photo-irradiated area becomes hydrophilic. Prior to the laser beam scanning experiment, the hydrophilic-hydrophobic contrast at the boundary between the photo-irradiated and the photo-masked area was examined. Figure 2(a) is a photograph of an OTS-treated paper immediately after being removed from the DMSO solution of CPI-410S. Since CPI-410S absorbs light of wavelength shorter than $410 \mathrm{~nm}$, the entire paper has a light-yellow color. The lower half of the paper was masked with black paper and illuminated 
with light from a xenon lamp. After photoirradiation for $50 \mathrm{~min}$, the photo-irradiated part and the photo-masked part were different in color as shown in Fig. 2(b). The photochemical reaction of triarylsulfonium salts is very complicated, and various reaction processes have been proposed in the literature. ${ }^{16}$ The heterolytic cleavage of triarylsulfonium gives diphenyl sulfide as shown in Fig. S1 (Supporting Information). Therefore, the color change indicates that the photolysis of CPI-410S proceeded in the upper part of the paper. After the photoirradiation, the paper was rinsed with chloroform and DMSO to wash out the CPI-410S, and both the upper and lower parts of the paper became colorless. To investigate the hydrophilic or hydrophobic properties, aqueous droplets containing Acid Red 1 were dropped on the upper and lower parts of the paper, respectively. As shown in Fig. 2(c), it was clearly visible that a hydrophilic-hydrophobic boundary was formed between the photo-irradiated and the photo-masked area. This result clearly indicates that the change from hydrophobic to hydrophilic nature of the OTS-treated paper was caused by the photochemical reaction with CPI-410S.

\section{Hydrophilic patterns fabricated on the OTS-treated paper using} the laser beam scanning technique

As described in the previous section, using CPI-410S as a photoacid generator, the photo-irradiated area on the OTStreated paper becomes hydrophilic. Therefore, by using a laser beam as a light source for the photochemical reaction and scanning the beam to draw an arbitrary spatial pattern on the paper, it should be possible to fabricate hydrophilic channels directly on the OTS-treated paper without using a photomask. To demonstrate this idea, using a computer-controlled Galvo mirror system, a 405-nm laser beam was scanned in a star pattern on the OTS-treated paper containing CPI-410S as shown

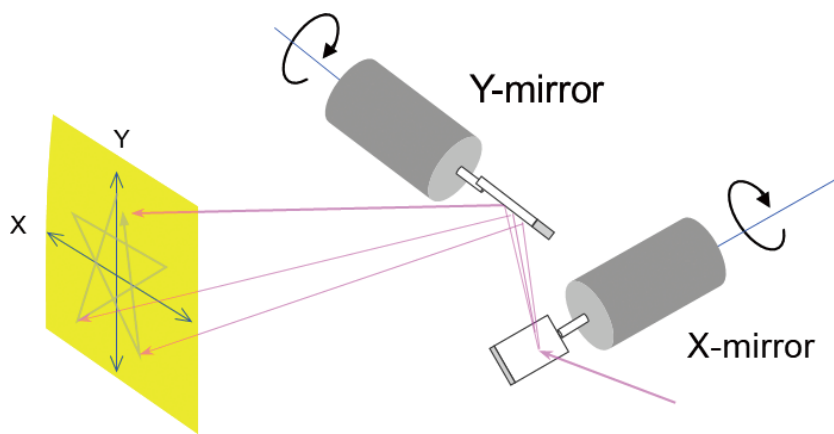

Fig. 3 Schematic illustration of a laser beam scanning with a Galvo mirror system. in Fig. 3. The continuous wave laser beam of $300 \mathrm{~mW}$ was loosely focused on the paper. The laser beam was scanned at a speed of $4.5 \mathrm{~m} / \mathrm{s}$, programmed to draw a star shape 90 times per second, and irradiated continuously on the paper for $50 \mathrm{~min}$. Then, the paper was rinsed with chloroform and DMSO to wash out the CPI-410S, and a pale-yellow star appeared on the paper (Fig. 4(a)). The width of the pale-yellow colored channel observed under an optical microscope was about $50 \mu \mathrm{m}$. When a water droplet containing Acid Red 1 was dropped on one vertex of the star, the aqueous solution spread along the channels via capillary forces as shown in Figs. 4(a) and 4(b). As shown in Fig. 4(c), the width of the red-colored line observed under an optical microscope was approximately $400 \mu \mathrm{m}$. This indicates that while the aqueous solution was flowing through the hydrophilic channel, it diffused in the lateral direction. To the best of our knowledge, this is the first report on the fabrication of hydrophilic channels on the OTS-treated paper using photoinduced acid generation processes coupled with the laser beam scanning technique.

\section{Quantitative analysis of nitrite}

Finally, the paper-based microfluidic device prepared by the present method was applied to the quantitative analysis of nitrite anion $\left(\mathrm{NO}_{2}^{-}\right)$using the Griess reaction, in which a red color appears due to the reaction of $\mathrm{NO}_{2}^{-}$with sulfanilamide and $N$-(1-naphthyl) ethylenediamine. ${ }^{17,18}$ As shown in Fig. 5, six linear hydrophilic channels were fabricated on the OTS-treated paper. An aqueous solution $(3 \mu \mathrm{L})$ containing $50 \mathrm{mM}$ sulfanilamide, $0.33 \mathrm{M}$ citric acid, and $10 \mathrm{mM} N$-(1-naphthyl) ethylenediamine sulfanilamide was permeated in each channel,

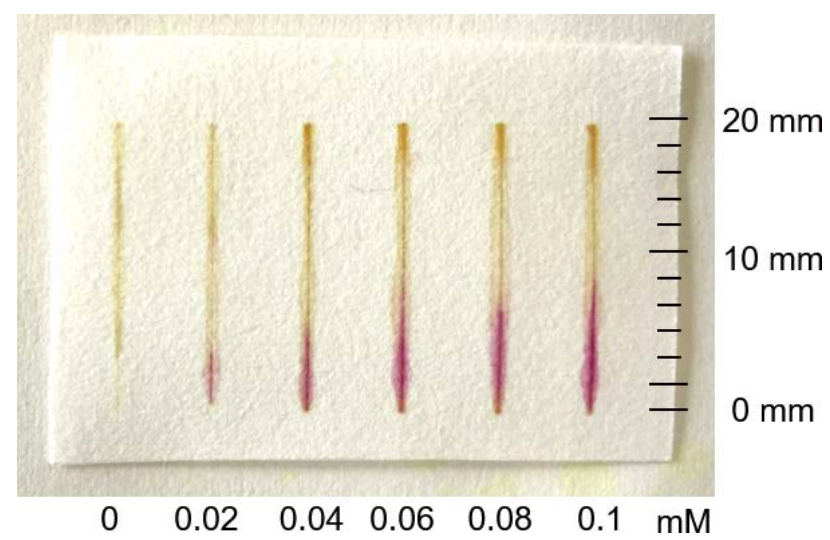

Fig. $5 \mathrm{NO}_{2}^{-}$concentration dependence of the length of the colored region.
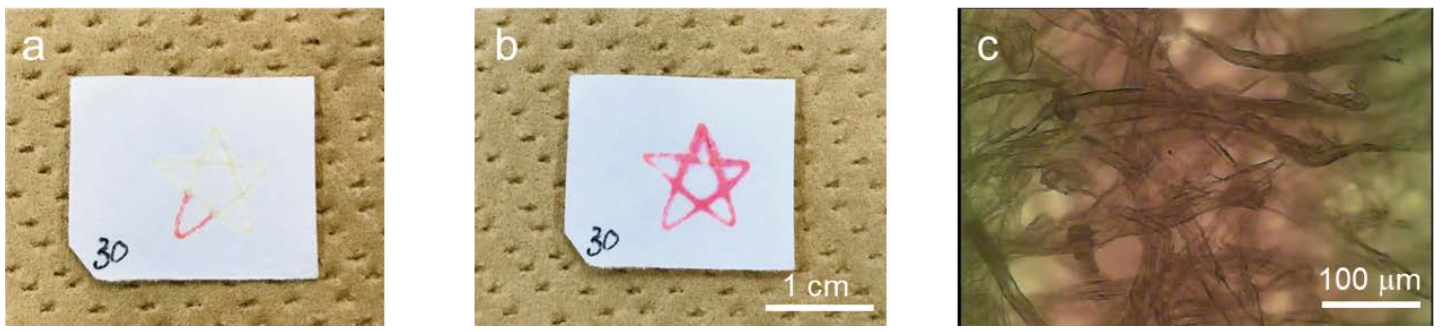

Fig. 4 The images display photographs of a star-shaped hydrophilic channel on the OTS-treated paper. An Acid Red 1 solution immersed in a part of the channel (a), in the entire channel (b), and the red-colored line observed under an optical microscope (c). 
and dried in an oven at $40^{\circ} \mathrm{C}$ for $10 \mathrm{~min}$. Standard solution was prepared by dissolving $\mathrm{NaNO}_{2}$ in water, and $0.25 \mu \mathrm{L}$ of standard solutions with different concentrations were dropped on the end of each channel. As shown in Fig. 5, the length of the red colored area increased with increasing $\mathrm{NO}_{2}^{-}$concentration. Although the color contrast at the forefront is slightly obscure, this preliminary experiment might suggest the possibility of quantitative analysis of $\mathrm{NO}_{2}^{-}$based on the length of the red colored area.

\section{Conclusions}

As demonstrated in this study, we achieved the fabrication of hydrophilic channels on the OTS-treated paper using photoinduced acid generation processes coupled with the laser beam scanning technique. Since the photoacid generators are used as photoinitiators for cationic polymerization, various photoacid generating compounds have been developed and widely used in industry so far. The method is very versatile, since similar experiments are expected to be possible with photoacid generators other than CPI-410S. Furthermore, since only the photo-irradiated area becomes hydrophilic, it would be possible to make paper-based microfluidic devices by irradiating light through a photomask on the OTS-treated papers in the presence of photoacid generators. In this study, we employed the laser beam scanning methods to create hydrophilic channels on the OTS-treated papers. Using the loosely focused laser beam as an excitation light source, the hydrophilic channels were successfully created on the paper in $50 \mu \mathrm{m}$ resolution. The minimum width of the hydrophilic channels approximately corresponds to the diameter of the spot of the laser beam on the paper. The channel width is one of the important factors governing the velocity of liquid flow via capillary forces. To improve the contrast of color change in the quantitative analysis of $\mathrm{NO}_{2}^{-}$, the flow rate dependence of the Griess reaction will be discussed in future studies. We are convinced that the photoinduced acid generation processes coupled with the laser beam scanning technique will be a versatile method to fabricate paperbased microfluidic devices.

\section{Acknowledgements}

We would like to thank San-Apro Ltd. for providing the CPI410S. This work was supported by the JSPS KAKENHI (grant numbers JP18H02006, JP18H03912, and JP19H04676).

\section{Supporting Information}

This material is available free of charge on the Web at http:// www.jsac.or.jp/analsci/.

\section{References}

1. A. W. Martinez, S. T. Phillips, M. J. Butte, and G. M. Whitesides, Angew. Chem., Int. Ed., 2007, 46, 1318.

2. D. M. Cate, J. A. Adkins, J. Mettakoonpitak, and C. S. Henry, Anal. Chem., 2015, 87, 19.

3. Y. Yang, E. Noviana, M. P. Nguyen, B. J. Geiss, D. S. Dandt, and C. S. Henry, Anal. Chem., 2017, 89, 71.

4. Y. He, Y. Wu, J. Fu, and W. Wu, RSC Adv., 2015, 5, 78109.

5. T. Akyazi, L. Basabe-Desmonts, and F. Benito-Lopez, Anal. Chim. Acta, 2018, 1001, 1.

6. C. L. Sones, I. N. Katis, P. J. W. He, B. Mills, M. F. Namiq, P. Shardlow, M. Ibsen, and R. W. Eason, Lab Chip, 2014, $14,4567$.

7. P. J. W. He, I. N. Katis, R. W. Eason, and C. L. Sones, Biomirofuidics, 2015, 9, 026503.

8. G. Chitnis, Z. Ding, C.-L. Chang, C. A. Savran, and B. Ziaie, Lab Chip, 2011, 11, 1161.

9. Q. He, C. Ma, X. Hu, and H. Chen, Anal. Chem., 2013, 85, 1327.

10. L. Zhang, H. Kwok, X. Li, and H.-Z. Yu, ACS Appl. Mater. Interfaces, 2017, 9, 39728.

11. Z. Tang, H. Li, D. W. Hess, and V. Breedveld, Cellulose, 2016, 23, 1401.

12. T. Kämäräinen, L. R. Arcot, L.-S. Johansson, J. Campbell, T. Tammelin, S. Franssila, J. Laine, and O. J. Rojas, Cellulose, 2016, 23, 1847.

13. L. Cai, C. Xu, S. Lin, J. Luo, M. Wu, and F. Yang, Biomicrofluidics, 2014, 8, 056504.

14. L. Cei, Y. Wang, Y. Wu, C. Xu, M. Zhong, H. Lai, and J. Huang, Analyst, 2014, 139, 4593.

15. A. Wolfberger, R. Kargl, T. Griesser, and S. Spirk, Molecules, 2014, 19, 16266.

16. J. L. Dektar and N. P. Hacker, J. Am. Chem. Soc., 1990, 112, 6004.

17. J. Sun, X. Zhang, M. Broderick, and H. Fein, Sensors, 2003, 3, 276.

18. D. Giustarini, R. Rossi, A. Milzani, and I. Dalle-Donne, Methods in Enzymology, 2008, 440, 361. 